\title{
Evolution of a Strategy for the Enantioselective Synthesis of (-)-Cajanusine
}

\author{
Renyu Guo, Brittany P. Witherspoon, and M. Kevin Brown*
}

Cite This: J. Am. Chem. Soc. 2020, 142, 5002-5006

Read Online

ABSTRACT: The first enantioselective synthesis of $(-)$-cajanusine is presented. Key features of the route include a rapid synthesis of the [4.2.0] bicyclooctane core by an enantioselective isomerization/stereoselective [2+2]-cycloaddition strategy as well as prominent use of catalytic methods for bond construction. The evolution of the approach is also presented that highlights unexpected roadblocks and how novel solutions were developed.

$\mathrm{T}^{\mathrm{n}}$ he stilbenoid oligomers constitute a broad family of natural products with high structural variance, many of which are derived from the common stilbenoid resveratrol. ${ }^{1}$ Most stilbenoid oligomers arise from radical-mediated cyclizations that lead to the formation of products with ring sizes five and greater. However, cyclobutane stilbenoid dimers that likely arise from a formal [2+2]-cycloaddition of the alkene units are known. ${ }^{1}$ In 2014, a new cyclobutanecontaining stilbenoid dimer, cajanusine (1), was reported. ${ }^{2}$ Here, the cyclobutane is likely generated from a formal $[2+2]$ cycloaddition with the oxygenated aryl group and the stilbenoid alkene unit, thus giving rise to a novel scaffold within this family of natural products (Scheme 1$)^{2}$

Scheme 1. Cajanusine and Biosynthetic Precursors

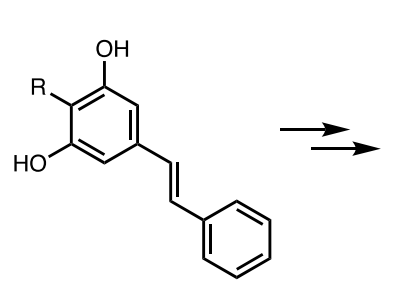

$\mathrm{R}=\mathrm{H}$ or prenyl

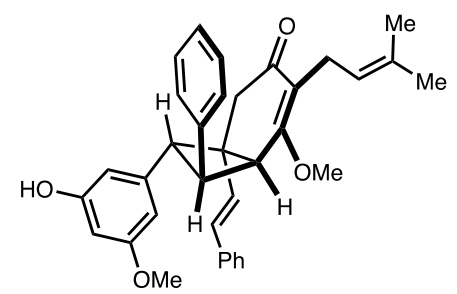

$(-)$-cajanusine (1)
Due to our laboratory's ongoing interest and work in developing enantioselective reactions to access cyclobutanes, we were drawn to targeting (-)-cajanusine (1) for synthesis. ${ }^{3}$ The pentasubstituted cyclobutane ring, as part of a highly functionalized [4.2.0] bicyclooctane core, was viewed as a challenge to access with traditional methods and strategies. Our group recently disclosed an enantioselective isomerization/stereoselective $[2+2]$-cycloaddition strategy for the synthesis of [4.2.0] bicyclooctanes. ${ }^{4-6}$ Given this premise, we focused on an approach that would implement this strategy toward (-)-cajanusine (1). As shown in Scheme 2, we expected that stereoselective $[2+2]$-cycloaddition of 2 would generate 3 . Since the stereochemistry at C7 is opposite that of the natural product, an epimerization would be necessary. Release of steric pressure of the syn-1,2-diarylcyclobutane to
Scheme 2. Initial Strategy for the Synthesis of Cajanusine

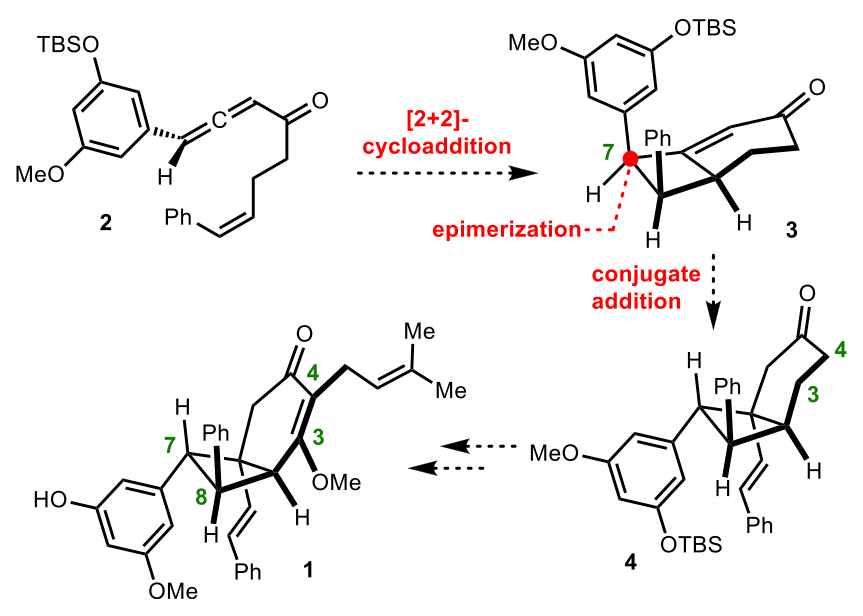

the anti-1,2-diarylcyclobutane would serve as a driving force for the reaction. Elaboration of $\mathbf{4}$ to $(-)$-cajanusine (1) was envisioned to occur through stereoselective conjugate addition, followed by oxidation and prenylation of the $\mathrm{C} 3$ and $\mathrm{C} 4$ positions, respectively.

Our initial efforts commenced with synthesis of $\beta, \gamma$ unsaturated alkynyl ketone $\mathbf{8}$, an intermediate necessary for the enantioselective isomerization/stereoselective $[2+2]$-cycloaddition process (Scheme 3). Cross-coupling of dihydropyran 5 with $\mathrm{PhMgBr}$ under $\mathrm{Ni}$ catalysis as described by Wenkert provided the desired alcohol, ${ }^{7}$ which was oxidized to the aldehyde under $\mathrm{Cu}$-catalyzed aerobic conditions reported by $\mathrm{Stahl}^{8}$ and then converted to epoxide 6. Addition of the

Received: January 11, 2020

Published: March 9, 2020 
Scheme 3. First-Generation Approach

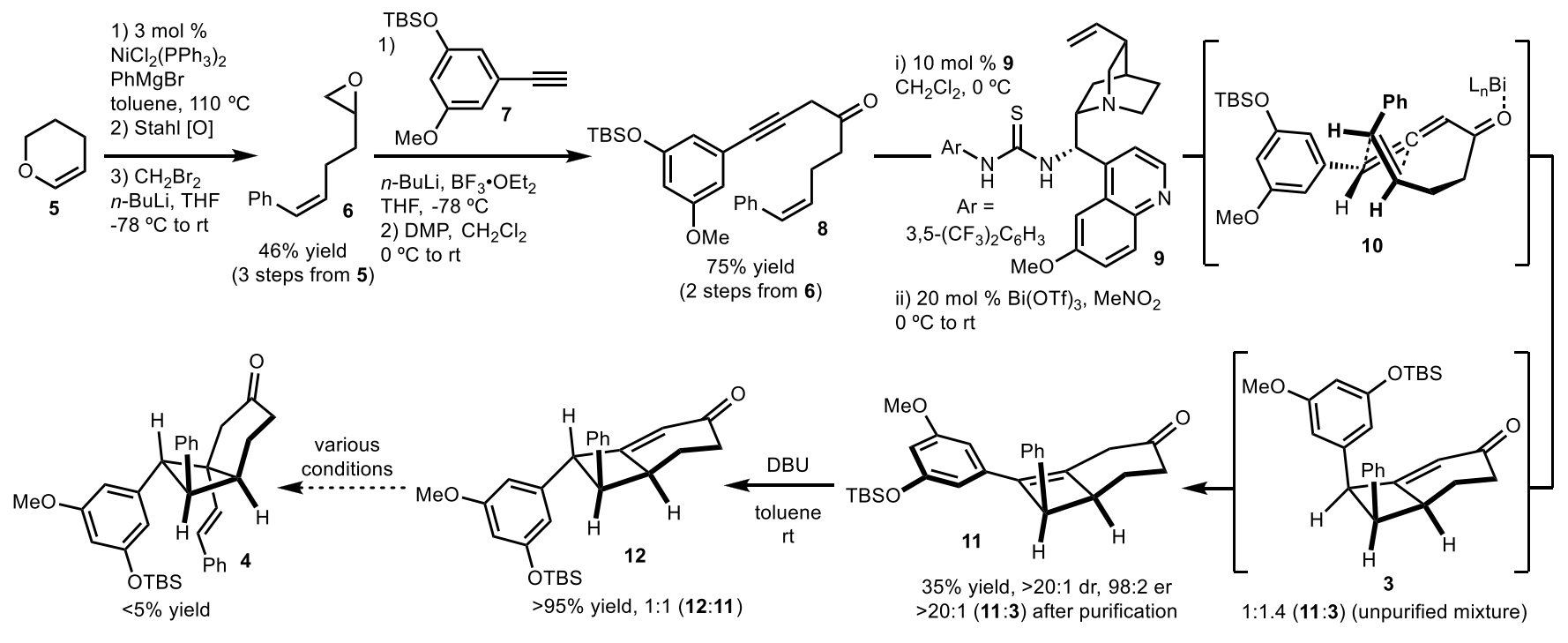

lithium acetylide of 7 and subsequent oxidation provided the $\beta, \gamma$-unsaturated alkynyl ketone 8 .

Enantioselective isomerization proceeded smoothly to generate the allenic ketone (not isolated), which, upon treatment with $\mathrm{Bi}(\mathrm{OTf})_{3}$, likely promoted the $[2+2]$-cycloaddition via pre-transition state $10{ }^{4}$ Examination of the unpurified reaction mixture by ${ }^{1} \mathrm{H}$ NMR revealed that, in addition to the expected product 3, cyclobutene 11 was formed as a significant product. ${ }^{4}$ Cyclobutene $\mathbf{1 1}$ likely arises from $\mathbf{3}$ under the reaction conditions via an acid-promoted alkene isomerization driven by relief of steric pressure of the syn-1,2diarylcyclobutane motif. Furthermore, any attempts at purification by chromatography resulted in complete isomerization to cyclobutene 11, which could be isolated in $35 \%$ yield and 98:2 er.

Given that epimerization at C7 is necessary for the synthesis of cajanusine, the isomerization reaction was deemed fortuitous. With access to cyclobutene 11, isomerization of the alkene into conjugation with the ketone with concomitant formation of the desired stereoisomer $\mathbf{1 2}$ (aryl groups now anti) could be achieved upon treatment with DBU (Scheme 3 ). However, it appears that $K_{\mathrm{eq}} \approx 1$, because the second isomerization event could not be driven toward completion under thermodynamic conditions. Preliminary ground-state calculations on the relative energies of $\mathbf{3}, \mathbf{1 1}$, and $\mathbf{1 2}$ gave them to be $1.98,0.00$, and $-0.04 \mathrm{kcal} / \mathrm{mol}$, respectively, which is consistent with experimental results. Despite the incomplete conversion to 12 , it was proposed that, after conjugate addition, cyclobutene $\mathbf{1 1}$ could be recycled if it was re-isolated from the reaction mixture. However, despite extensive experimentation, conjugate addition of a styrenyl-derived nucleophile to $\mathbf{1 2}$ could not be achieved. This is likely due to the steric demands in the generation of a quaternary carbon adjacent to the aryl group. This obstacle, coupled with the incomplete isomerization to $\mathbf{1 2}$, which limited material throughput, forced us to consider other synthetic strategies toward (-)-cajanusine.

A second-generation approach was devised based on the premise that much of the difficulties experienced were due to an early-stage incorporation of the aryl group at C7 that forced a challenging conjugate addition. Therefore, strategies that installed the aryl group subsequent to the conjugate addition were investigated (Scheme 4). Since the enantioselective isomerization/stereoselective $[2+2]$-cycloaddition process

Scheme 4. Second-Generation Approach<smiles>[R]C=C=CC(=O)CCC=Cc1ccccc1</smiles><smiles></smiles>

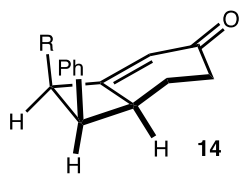

conjugate addition
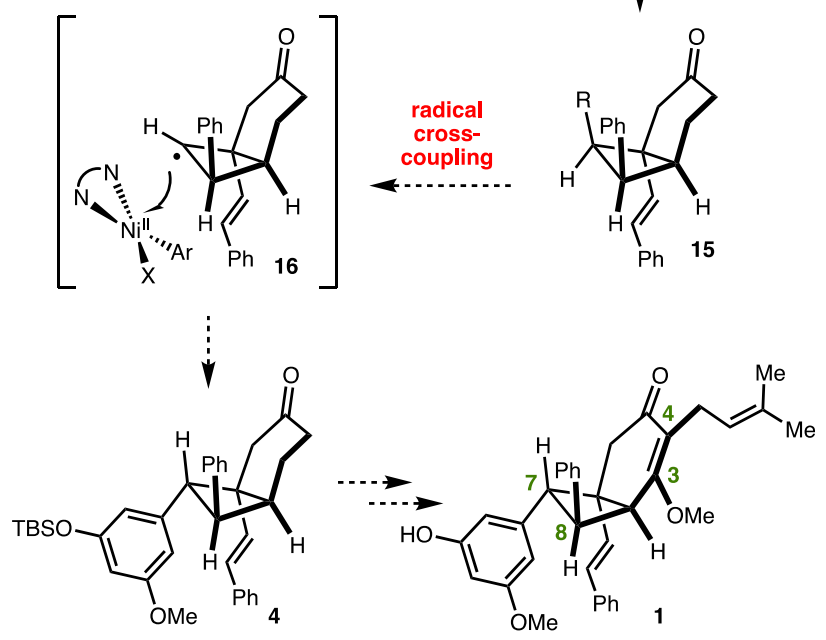

gives rise to the syn-diastereomer 14, the aryl group at C7 needed to be incorporated from a functional handle that allowed for stereoselective $\mathrm{C}-\mathrm{C}$ bond formation. With these criteria in mind, we considered a cross-coupling approach involving formation of a radical at C7 (16) that would allow for capture to occur on the convex face of the [4.2.0]bicyclooctane core. Given the advances in Ni-catalyzed crosscoupling that involve the intermediacy of alkyl radicals, this strategy was deemed promising. ${ }^{9}$

With the consideration that an alkyl halide or redox-active ester would be necessary for the installation of the aryl group via $\mathrm{Ni}$-catalyzed cross-coupling, a versatile protected alcohol was used as a handle. The requisite $\beta, \gamma$-alkynyl ketone 17 was 

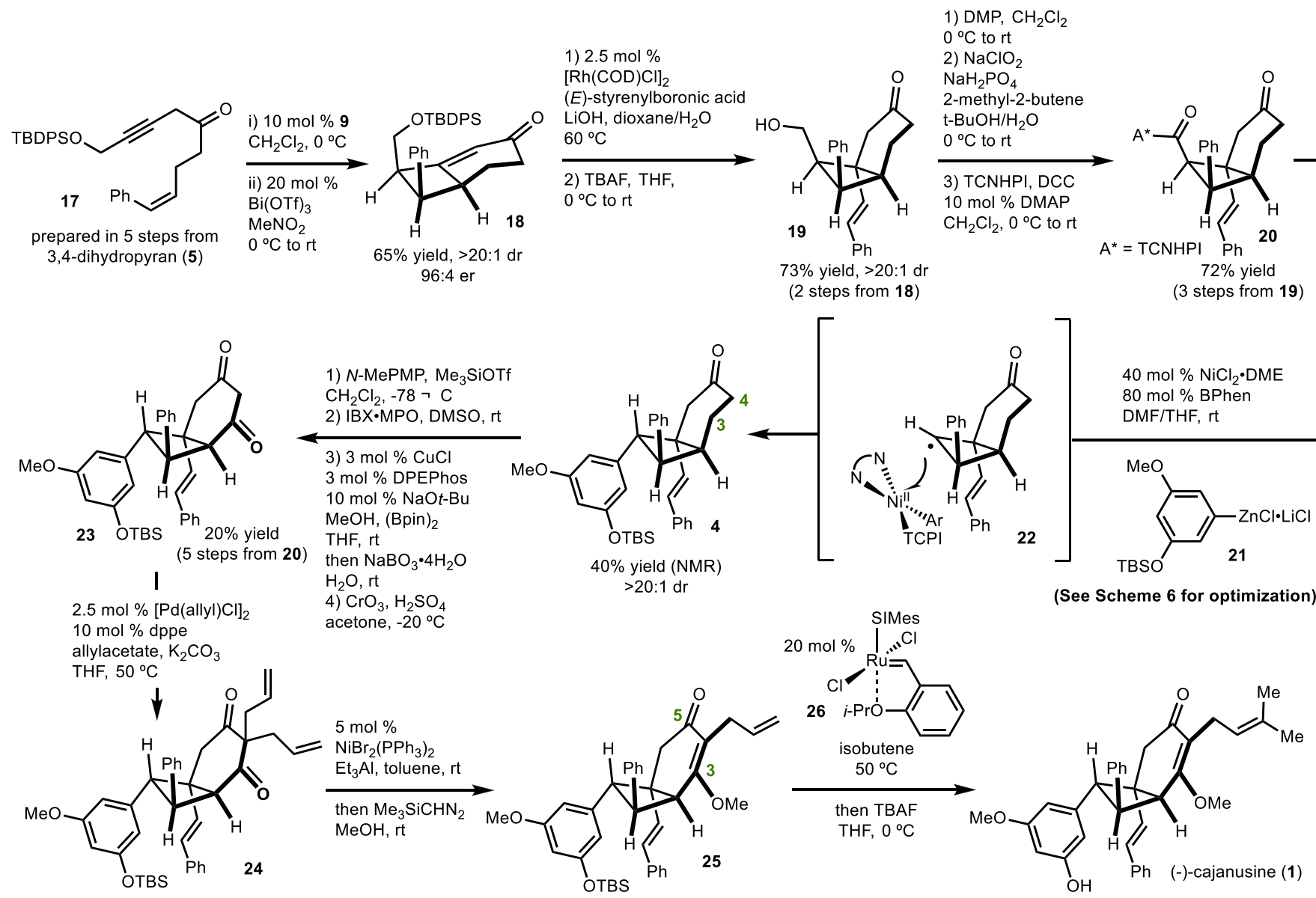

$98 \%$ yield

acetone, $-20{ }^{\circ} \mathrm{C}$

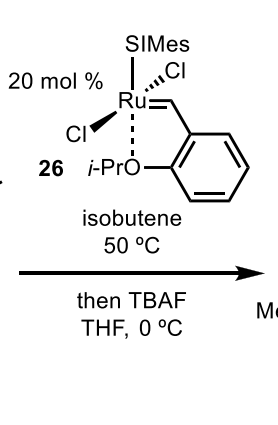

(See Scheme 6 for optimization)

$80 \mathrm{~mol} \%$ BPhen

DMF/THF, rt

prepared in a sequence analogous to that shown in Scheme 3 in five steps (Scheme 5). ${ }^{10}$ The enantioselective isomerization/ stereoselective $[2+2]$-cycloaddition sequence proceeded to form 18 in good yield, diastereoselectivity, and enantioselectivity. ${ }^{4}$ Conjugate addition of E-styrenylboronic acid could be achieved through the use of $\mathrm{Rh}$ catalysis which, after desilylation, provided 19 in $73 \%$ yield. ${ }^{11}$ Other approaches involving cuprates proved less fruitful and resulted in the formation of several unidentified impurities.

We elected to explore incorporation of the aryl group by cross-coupling of a redox-active ester $(\mathbf{2 0}, \mathbf{2 7})$, which could be prepared from 19 by oxidation to the acid and coupling with the necessary reagent. As has been noted, cross-coupling reactions of redox-active esters are substrate dependent, and it is therefore difficult to predict the success of any given set of conditions. $^{12 a}$ Therefore, an initial evaluation of 18 crosscoupling reactions ${ }^{12}$ and 8 reductive cross-coupling reactions ${ }^{13}$ of various redox-active esters $(\mathbf{2 0}, \mathbf{2 7}$, and 28$)$ was conducted (Scheme 6). ${ }^{10}$ In general, the reductive cross-coupling reactions returned mostly starting material, whereas the standard cross-coupling reactions consumed the starting material. Of the 26 reactions evaluated, only a Ni-catalyzed cross-coupling $^{14}$ of arylzinc 21 and an Fe-catalyzed crosscoupling $^{15}$ of diarylzinc reagent 29 , both with redox-active ester 20 (TCNHPI = tetrachloro- $N$-hydroxyphthalimide), delivered the product in promising yields (20\% and $11 \%$, respectively). The Ni-catalyzed cross-coupling was selected for further optimization because of the higher initial yield (Scheme
6 , inset table, entry 1$)$. A screen of ligands revealed that BPhen was superior to dtbpy (Scheme 6, inset table, entry 5). ${ }^{16}$ While further improvement in yield was not observed with other temperatures or increased quantities of arylzinc reagent $\mathbf{2 1}$ (Scheme 6, inset table entries 6-8), higher catalyst loadings did result in increased yield (Scheme 6, inset table entry 9).

At this stage, completion of the synthesis required incorporation of the substituents at $\mathrm{C} 4$ and $\mathrm{C} 3$. This sequence was initiated through selective enolization of the sterically less demanding $\alpha$-position of the ketone with $N$-MePMP $(N$ methyl pentamethylpiperdine) and $\mathrm{Me}_{3} \mathrm{SiOTf}$ (Scheme 5). ${ }^{17}$ It is important to note that deprotonation with LDA, LHMDS, $\mathrm{LiTMP}$, or $\mathrm{LiN}(t-\mathrm{Bu}) \mathrm{CPh}_{3}{ }^{18}$ resulted in the formation of enolate isomers. Conversion of the enol silane to the $\alpha, \beta$ unsaturated carbonyl was achieved by treatment with IBX. MPO. ${ }^{19}$ Copper-catalyzed conjugate addition of Bpin and subsequent oxidation resulted in the formation of dione $\mathbf{2 3}$ (1:2:1 mixture of keto and enol tautomers $){ }^{20}$ Direct installation of the prenyl group proved challenging, as has been noted in the syntheses of other prenylated natural products. $^{21}$ Therefore, an approach involving allylation followed by cross-metathesis with isobutene was explored. Surprisingly, it was difficult to incorporate a single allyl group, as bis-allylation was difficult to suppress. Thus, an efficient twostep sequence was developed in which Pd-catalyzed bisallylation $^{22}$ was followed by Ni-catalyzed mono-deallylation. ${ }^{23}$ Finally, regioselective O-methylation (4:1 C3-OMe versus C5$\mathrm{OMe},>20: 1$ after purification), cross-metathesis ${ }^{24}$ with 


\section{Scheme 6. Optimization of the Cross-Coupling}

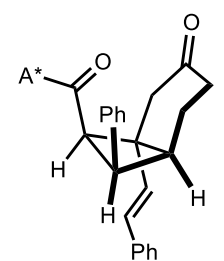

$$
\begin{gathered}
18 \text { cross-coupling reactions } \\
\text { and } \\
8 \text { reductive cross-coupling } \\
\text { reactions }
\end{gathered}
$$

initial screen of (see SI for details)

$$
\mathrm{A}^{*}=\text { TCNHPI (20) }
$$

\begin{tabular}{|c|c|}
\hline $\begin{array}{c}\mathrm{A}^{*}=\mathrm{TCNHPI} \\
20 \mathrm{~mol} \% \mathrm{NiCl}_{2} \cdot \mathrm{DME} \\
40 \mathrm{~mol} \% \mathrm{dtbpy} \\
3.0 \text { equiv } \mathrm{ArZnCl} \cdot \mathrm{LiCl}(\mathbf{2 1}) \\
\text { DMF/THF, rt }\end{array}$ & $\begin{array}{c}\mathrm{A}^{*}=\mathrm{TCNHPI} \\
10 \mathrm{~mol} \% \mathrm{Fe}(\mathrm{acac})_{3} \\
12 \mathrm{~mol} \% \mathrm{dppBz} \\
3.0 \text { equiv } \mathrm{Ar}_{2} \mathrm{Zn} \mathrm{(29)} \\
\text { THF, rt }\end{array}$ \\
\hline $20 \%$ yield, $>20: 1 d r$ & $11 \%$ yield, $>20: 1 d r$ \\
\hline
\end{tabular}
$A^{*}=\operatorname{NHPI}(27)$ $\mathrm{A}^{*}=\mathrm{OH}(\mathbf{2 8})$ (in situ activation)

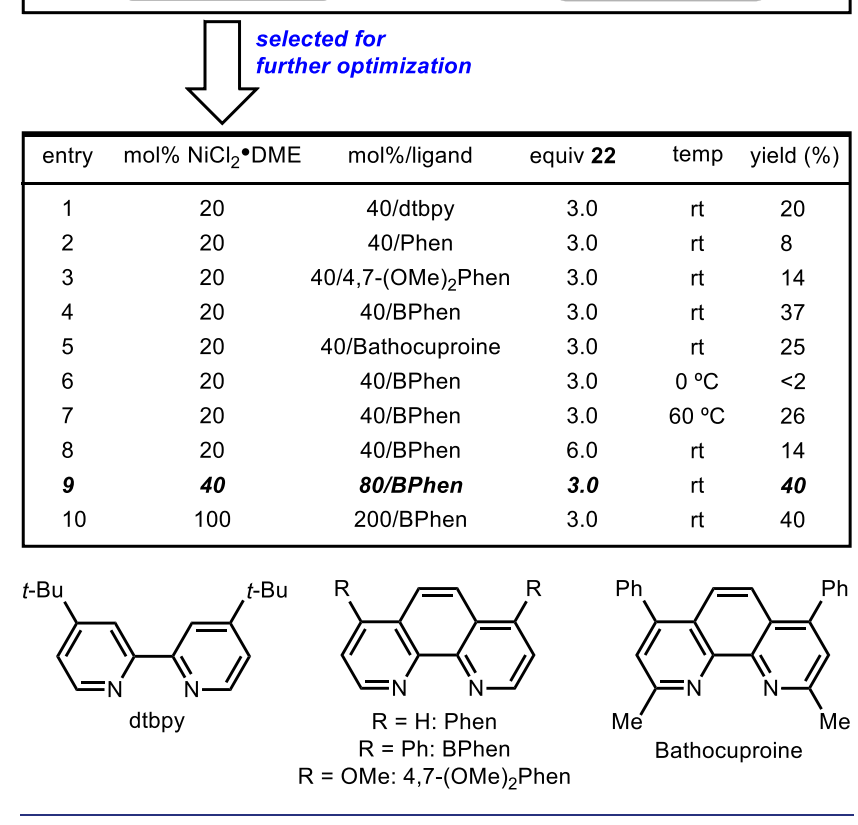

isobutene, and deprotection allowed for synthesis of $(-)$-cajanusine $(\mathbf{1})$. Due to the scalability and simplicity of many of the steps, $>100 \mathrm{mg}$ of cajanusine was easily prepared (98 $\mathrm{mg}$ was prepared in one reaction).

In summary, the first enantioselective synthesis of (-)-cajanusine is presented. The evolution of the strategy is highlighted to showcase the unexpected challenges that occurred and the solutions that were developed. In addition, the synthesis demonstrates the utility of various catalytic methods to function in complex settings and further underscores the utility of the enantioselective isomerization/ stereoselective $[2+2]$-cycloaddition method developed in our laboratory.

\section{ASSOCIATED CONTENT}

\section{SI Supporting Information}

The Supporting Information is available free of charge at https://pubs.acs.org/doi/10.1021/jacs.0c00359.

Experimental procedures; analytical data for all new compounds (PDF)

\section{AUTHOR INFORMATION}

\section{Corresponding Author}

M. Kevin Brown - Department of Chemistry, Indiana University, Bloomington, Indiana 47405, United States; 이이.org/0000-0002-4993-0917; Email: brownmkb@ indiana.edu

\section{Authors}

Renyu Guo - Department of Chemistry, Indiana University, Bloomington, Indiana 47405, United States

Brittany P. Witherspoon - Department of Chemistry, Indiana University, Bloomington, Indiana 47405, United States

Complete contact information is available at: https://pubs.acs.org/10.1021/jacs.0c00359

\section{Notes}

The authors declare no competing financial interest.

\section{ACKNOWLEDGMENTS}

We thank Indiana University and the NIH (R01GM110131 and R35GM131755) for financial support. This project was partially funded by the Vice Provost for Research through the Research Equipment Fund and the NSF (CHE1726633). We thank Qi Cui (Peking University) for calculations on intermediates 3, 11, and 12. We thank Prof. Ying Wang (Jinan University) for providing a sample of cajanusine.

\section{REFERENCES}

(1) Shen, T.; Wang, X.-N.; Lou, H.-X. Natural Stilbenes: an Overview. Nat. Prod. Rep. 2009, 26, 916.

(2) Li, X.-L.; Zhao, B.-X.; Huang, X.-J.; Zhang, D.-M.; Jiang, R.-W.; Li, Y.-J.; Jian, Y.-Q.; Wang, Y.; Li, Y.-L.; Ye, W.-C. (+)- And (-)-Cajanusine, a Pair of New Enantiomeric Stilbene Dimers with a New Skeleton From the Leaves of Cajanus Cajan. Org. Lett. 2014, 16, 224.

(3) (a) Conner, M. L.; Xu, Y.; Brown, M. K. Catalytic Enantioselective Allenoate-Alkene [2+2] Cycloadditions. J. Am. Chem. Soc. 2015, 137, 3482-3485. (b) Xu, Y.; Hong, Y. J.; Tantillo, D. J.; Brown, M. K. Intramolecular Chirality Transfer [2+2] Cycloadditions of Allenoates and Alkenes. Org. Lett. 2017, 19, 3703-3706. (c) Wiest, J. M.; Conner, M. L.; Brown, M. K. Synthesis of (-)-Hebelophyllene-E: an Entry to Geminal Dimethyl-Cyclobutanes by [2+2] Cycloaddition of Alkenes and Allenoates. Angew. Chem., Int. Ed. 2018, 57, 4647-4651. (d) Wiest, J. M.; Conner, M. L.; Brown, M. K. Allenoates in Enantioselective [2+2] Cycloadditions: From a Mechanistic Curiosity to a Stereospecific Transformation. J. Am. Chem. Soc. 2018, 140, 15943-15949. (e) Conner, M. L.; Wiest, J. M.; Brown, M. K. Thioallenoates in Catalytic Enantioselective [2+2]Cycloadditions with Unactivated Alkenes. Tetrahedron 2019, 75, 3265-3271.

(4) Line, N. J.; Witherspoon, B. P.; Hancock, E. N.; Brown, M. K. Synthesis of Ent-[3]-Ladderanol: Development and Application of Intramolecular Chirality Transfer [2+2] Cycloadditions of Allenic Ketones and Alkenes. J. Am. Chem. Soc. 2017, 139, 14392.

(5) For $[2+2]$-cycloaddition with allenic ketones, see: Zhao, J.-F.; Loh, T.-P. Acid-Catalyzed Intramolecular $[2+2]$ Cycloaddition of Ene-Allenones: Facile Access to Bicyclo [N.2.0] Frameworks. Angew. Chem., Int. Ed. 2009, 48, 7232.

(6) For enantioselective isomerization to access allenoates, see: (a) Liu, H.; Leow, D.; Huang, K.-W.; Tan, C.-H. Enantioselective Synthesis of Chiral Allenoates by Guanidine-Catalyzed Isomerization of 3-Alkynoates. J. Am. Chem. Soc. 2009, 131, 7212. (b) Inokuma, T.; Furukawa, M.; Uno, T.; Suzuki, Y.; Yoshida, K.; Yano, Y.; Matsuzaki, K.; Takemoto, Y. Bifunctional Hydrogen-Bond Donors That Bear a Quinazoline or Benzothiadiazine Skeleton for Asymmetric Organocatalysis. Chem. - Eur. J. 2011, 17, 10470. 
(7) Wenkert, E.; Michelotti, E. L.; Swindell, C. S.; Tingoli, M. Transformation of Carbon-Oxygen Into Carbon-Carbon Bonds Mediated by Low-Valent Nickel Species. J. Org. Chem. 1984, 49, 4894.

(8) Hoover, J. M.; Stahl, S. S. Highly Practical Copper(I)/TEMPO Catalyst System for Chemoselective Aerobic Oxidation of Primary Alcohols. J. Am. Chem. Soc. 2011, 133, 16901.

(9) Hu, X. Nickel-Catalyzed Cross Coupling of Non-Activated Alkyl Halides: a Mechanistic Perspective. Chem. Sci. 2011, 2, 1867.

(10) See the Supporting Information for details.

(11) Hayashi, T.; Yamasaki, K. Rhodium-Catalyzed Asymmetric 1,4Addition and Its Related Asymmetric Reactions. Chem. Rev. 2003, 103, 2829.

(12) (a) Sandfort, F.; O’Neill, M. J.; Cornella, J.; Wimmer, L.; Baran, P. S. Alkyl-(Hetero)Aryl Bond Formation via Decarboxylative CrossCoupling: a Systematic Analysis. Angew. Chem., Int. Ed. 2017, 56, 3319. (b) Liu, X.-G.; Zhou, C.-J.; Lin, E.; Han, X.-L.; Zhang, S.-S.; Li, Q; Wang, H. Decarboxylative Negishi Coupling of Redox-Active Aliphatic Esters by Cobalt Catalysis. Angew. Chem., Int. Ed. 2018, 57, 13096. (c) Chen, T.-G.; Zhang, H.; Mykhailiuk, P. K.; Merchant, R. R.; Smith, C. A.; Qin, T.; Baran, P. S. Quaternary Centers by NickelCatalyzed Cross-Coupling of Tertiary Carboxylic Acids and (Hetero)Aryl Zinc Reagents. Angew. Chem., Int. Ed. 2019, 58, 2454.

(13) (a) Huihui, K. M. M.; Caputo, J. A.; Melchor, Z.; Olivares, A. M.; Spiewak, A. M.; Johnson, K. A.; Dibenedetto, T. A.; Kim, S.; Ackerman, L. K. G.; Weix, D. J. Decarboxylative Cross-Electrophile Coupling of $\mathrm{N}$-Hydroxyphthalimide Esters with Aryl Iodides. J. Am. Chem. Soc. 2016, 138, 5016. (b) Anka-Lufford, L. L.; Huihui, K. M. M.; Gower, N. J.; Ackerman, L. K. G.; Weix, D. J. Nickel-Catalyzed Cross-Electrophile Coupling with Organic Reductants in Non-Amide Solvents. Chem. - Eur. J. 2016, 22, 11564. (c) Suzuki, N.; Hofstra, J. L.; Poremba, K. E.; Reisman, S. E. Nickel-Catalyzed Enantioselective Cross-Coupling of $\mathrm{N}$-Hydroxyphthalimide Esters with Vinyl Bromides. Org. Lett. 2017, 19, 2150. (d) Zhang, J.; Li, Z.; Zhuo, J.; Cui, Y.; Han, T.; Li, C. Tandem Decarboxylative Cyclization/Alkenylation Strategy for Total Syntheses of (+)-Longirabdiol, (-)-Longirabdolactone, and (-)-Effusin. J. Am. Chem. Soc. 2019, 141, 8372.

(14) (a) Cornella, J.; Edwards, J. T.; Qin, T.; Kawamura, S.; Wang, J.; Pan, C.-M.; Gianatassio, R.; Schmidt, M.; Eastgate, M. D.; Baran, P. S. Practical Ni-Catalyzed Aryl-Alkyl Cross-Coupling of Secondary Redox-Active Esters. J. Am. Chem. Soc. 2016, 138, 2174. (b) Beck, J. C.; Lacker, C. R.; Chapman, L. M.; Reisman, S. E. A Modular Approach to Prepare Enantioenriched Cyclobutanes: Synthesis of (+)-Rumphellaone A. Chem. Sci. 2019, 10, 2315. (c) Shang, M.; Feu, K. S.; Vantourout, J. C.; Barton, L. M.; Osswald, H. L.; Kato, N.; Gagaring, K.; McNamara, C. W.; Chen, G.; Hu, L.; Ni, S.; FernándezCanelas, P.; Chen, M.; Merchant, R. R.; Qin, T.; Schreiber, S. L.; Melillo, B.; Yu, J.-Q.; Baran, P. S. Modular, Stereocontrolled C $\beta$-H/ $\mathrm{C} \alpha$-C Activation of Alkyl Carboxylic Acids. Proc. Natl. Acad. Sci. U. S. A. 2019, 116, 8721.

(15) Toriyama, F.; Cornella, J.; Wimmer, L.; chen, T.-G.; Dixon, D. D.; Creech, G.; Baran, P. S. Redox-Active Esters in Fe-Catalyzed C-C Coupling. J. Am. Chem. Soc. 2016, 138, 11132.

(16) See the Supporting Information of ref 14a.

(17) Hu, P.; Chi, H. M.; DeBacker, K. C.; Gong, X.; Keim, J. H.; Hsu, I. T.; Snyder, S. A. Quaternary-Centre-Guided Synthesis of Complex Polycyclic Terpenes. Nature 2019, 569, 703.

(18) Busch-Petersen, J.; Corey, E. J. Sterically Shielded Secondary $\mathrm{N}$-Tritylamines and N-Tritylamide Bases, Readily Available and Useful Synthetic Reagents. Tetrahedron Lett. 2000, 41, 2515.

(19) Nicolaou, K. C.; Gray, D. L. F.; Montagnon, T.; Harrison, S. T. Oxidation of Silyl Enol Ethers by Using IBX and IBX $\bullet N-O x i d e$ Complexes: a Mild and Selective Reaction for the Synthesis of Enones. Angew. Chem., Int. Ed. 2002, 41, 996.

(20) Mun, S.; Lee, J.-E.; Yun, J. Copper-Catalyzed $\beta$-Boration of $\alpha$, $\beta$-Unsaturated Carbonyl Compounds: Rate Acceleration by Alcohol Additives. Org. Lett. 2006, 8, 4887.
(21) For a review, see: Yang, X.-W.; Grossman, R. B.; Xu, G. Research Progress of Polycyclic Polyprenylated Acylphloroglucinols. Chem. Rev. 2018, 118, 3508.

(22) Ruprah, P. K.; Cros, J.-P.; Pease, J. E.; Whittingham, W. G; Williams, J. M. J. Studies Towards the Total Synthesis of Cycloaraneosene and Ophiobolin M: A General Strategy for the Construction of the 5-8 Bicyclic Ring System. Eur. J. Org. Chem. 2002, 2002, 3145.

(23) Nečas, D.; Turský, M.; Kotora, M. Catalytic Deallylation of Allyl- and Diallylmalonates. J. Am. Chem. Soc. 2004, 126, 10222.

(24) Garber, S. B.; Kingsbury, J. S.; Gray, B. L.; Hoveyda, A. H. Efficient and Recyclable Monomeric and Dendritic Ru-Based Metathesis Catalysts. J. Am. Chem. Soc. 2000, 122, 8168. 